\title{
Selective ion sensors based on ionophore-modified graphene field-effect transistors
}

\author{
Kenzo Maehashi, Yasuyuki Sofue, Shogo Okamoto, Yasuhide Ohno, Koichi Inoue and \\ Kazuhiko Matsumoto \\ The Institute of Scientific and Industrial Research, Osaka University \\ 8-1 Mihogaoka, Ibaraki, Osaka 567-0047, Japan \\ E-mail: maehashi@sanken.osaka-u.ac.jp
}

\begin{abstract}
Selective potassium $(K)$ ion sensors were fabricated by modifying graphene field-effect transistors (FETs) with valinomycin, a selective $\mathrm{K}$ ionophore. The valinomycin-modified graphene FETs demonstrated highly sensitive, selective electrical detection of $\mathrm{K}$ ions in electrolytes. The $\mathrm{K}$ ions bound to the valinomycin in the graphene channel and affected the electrical potential of the channel. The transfer curves were shifted in a negative direction as the $\mathrm{K}$ ion concentration increased, indicating that $\mathrm{K}$ ions in solution were effectively detected over a wide concentration range, from $10 \mathrm{nM}$ to 1.0 $\mathrm{mM}$. The addition of $\mathrm{Na}$ ions did not cause any change in the transfer characteristics. We have thus demonstrated the utility of graphene FETs as highly sensitive, selective $\mathrm{K}$ ion sensors.
\end{abstract}

Key words: graphene field-effect transistors, selective ion sensors, potassium, ionophore, valinomycin

\section{Introduction}

A highly sensitive detection of biomolecules or chemical molecules has recently attracted much attention for various applications. Labelfree electrical monitoring of biorecognition events provides a promising platform, which is simpler, less expensive and requires less energy. Rapid testing of different proteins is required in various applications, including clinical diagnostics, environmental testing, food analysis, bioterrorism detection technologies, etc [1-5].

Graphene is a two-dimensional material [6]. Electrical characteristics in graphene field-effect transistors (FETs) are expected to be very sensitive for modulation of surface potentials in graphene channels [7]. Thus, graphene FETs are attractive as electric-readout biological, or chemical sensors owing to their superior properties such as high sensitivity, high conductance, and fast response [8-11]. In this paper, we have fabricated graphene FET-based chemical sensors with high sensitivity. Moreover, we report the integration of valinomycin into graphene FETs to achieve ion selectivity. Valinomycin has a high affinity for $\mathrm{K}$ relative to other alkali metal ions $[12,13]$. We report the fabrication and electrical characterization of valinomycin-modified graphene FET-based chemical sensors, and the investigation of their selective detection of $\mathrm{K}$ ions. The valinomycin-modified graphene FETs responded differently to $\mathrm{K}$ and $\mathrm{Na}$ ions over a wide concentration range, demonstrating their utility for monitoring $\mathrm{K}$ ions in solutions.
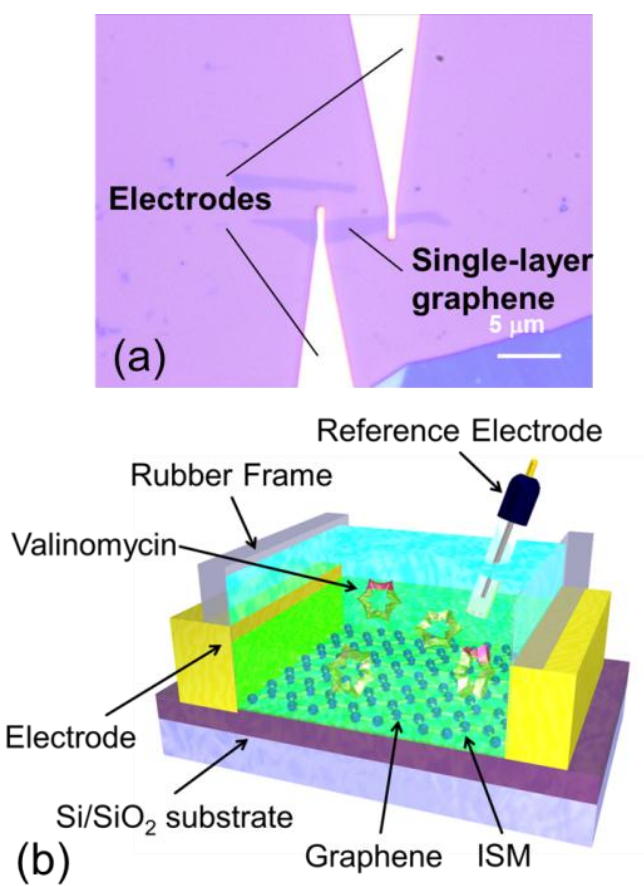

Fig. 1. (a) Scanning electron microscope image of a graphene-based FET and (b) schematic image of a measurement system. 


\section{Experimental}

A graphene was obtained by mechanical exfoliation. Only monolayer graphene was used for the channel to obtain high sensitivity. The number of graphene layer was confirmed by Raman spectroscopy [14,15]. Figure 1(a) shows graphene FETs fabricated by e-beam lithography and lift-off method on a $\mathrm{SiO}_{2}$ layer. Au was used for source and drain electrodes.

Figure 1(b) shows a schematic image of a measurement system. A silicone rubber was placed on the graphene FET so that the graphene channel was immersed in a buffer solution. An $\mathrm{Ag} / \mathrm{AgCl}$ reference electrode was used as the top-gated electrode to minimise environmental effects $[16,17]$. To achieve selective detection, the graphene channels were covered with ion selective membrane (ISM), constructed from a mixture of polyvinyl chloride and valinomycin. The electrical characteristics in the graphene FET were measured with a semiconductor parameter analyzer. All experiments were carried out in a supporting electrolyte of tris-(hydroxymethyl)aminomethane and $\mathrm{HCl}$ (Tris- $\mathrm{HCl}$ ) buffer solution $(0.1 \mathrm{M}, \mathrm{pH} 8.6)$ at room temperature. $\mathrm{KCl}$ solutions of various concentrations were added to the Tris- $\mathrm{HCl}$ buffer solution to increase the $\mathrm{K}$ ion concentration in the reagent solution.

\section{Transfer characteristics in a solution}
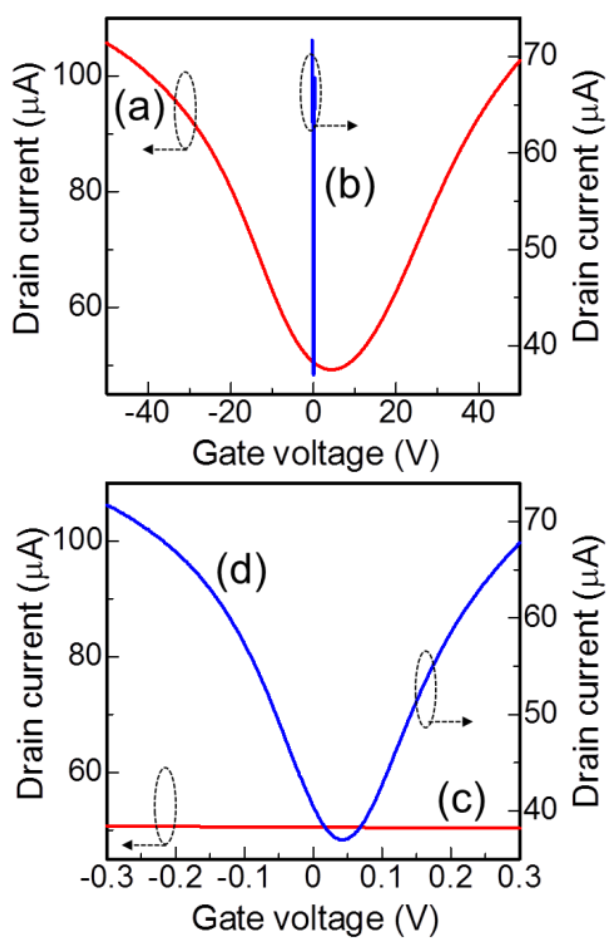

Fig. 2. Transfer characteristics in grapheme FET against back-gate voltage in vacuum (a) and top-gate voltage in a solution. (c) and (d) show the enlarged view of (a) and (b), respectively.
Figure 2 shows transfer characteristics in the graphene FET at room temperature. A typical ambipolar characteristic against back-gated voltage was observed in vacuum, as shown in Fig. 2(a). The transconductance was estimated to be $1.8 \mu \mathrm{S}$ in a vacuum. The drain current in the graphene FETs was plotted against the topgate voltage in an electrolyte, as shown in Fig. 2(b). Figures 2(c) and 2(d) show the enlarged view of Figs. 2(a) and 2(b), respectively, which were obtained by sweeping from -0.30 to $0.30 \mathrm{~V}$ of the gate voltage. The transconductance in the top-gated graphene FET was estimated to be $190 \mu \mathrm{S}$, which is 100 -fold larger than that in vacuum. This indicates that the electrical double layers act as thin insulators $[18,19]$. As a result, graphene FETs are useful to detect chemical and biological molecules with high sensitivity.

\section{Selective ion sensors}

Figure 3 shows transfer characteristics of valinomycin-modified graphene FETs in the 100 $\mathrm{mM}$ Tris- $\mathrm{HCl}$ buffer solution with various $\mathrm{KCl}$ concentrations over the range from 0 to $1.0 \mathrm{mM}$. With increasing $\mathrm{K}$ ion concentration, the topgate voltage at the Dirac point shifted toward negative direction, which is due to the accumulation of $\mathrm{K}$ ions caused by valinomycin on the graphene surfaces. The electrostatic potential of graphene surfaces exhibit that the $\mathrm{K}$ ion concentration dependence of the top-gate voltage at the Dirac point of valinomycinmodified graphene FETs is roughly linear on a semilogarithmic scale, indicating an equilibrium between the $\mathrm{K}$ ions in the valinomycin sites and in solution. These results show valinomycinmodified graphene FETs detected $\mathrm{K}$ ions with concentration from $10 \mathrm{nM}$ to $1.0 \mathrm{mM}$.

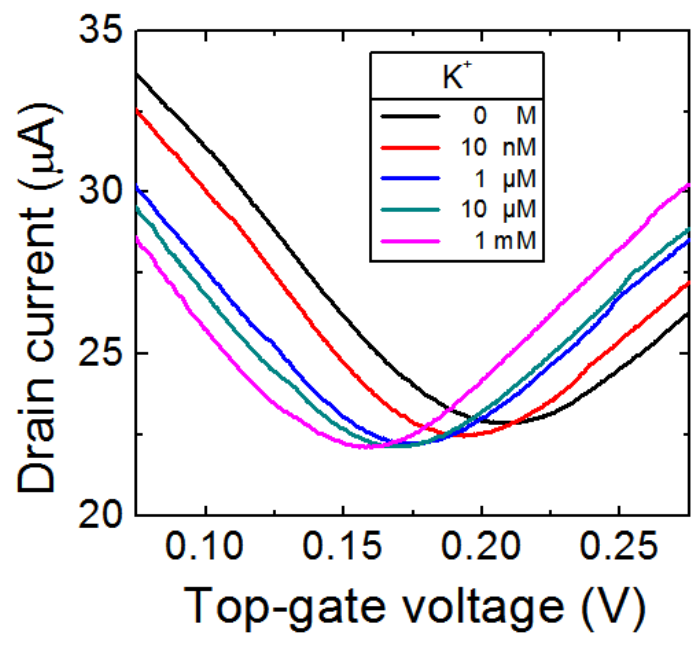

Fig. 3. Transfer characteristics of a valinomycinmodified graphene FET in the $100 \mathrm{mM}$ Tris- $\mathrm{HCl}$ buffer solution with various $K$ ion concentrations over the range from 0 to $1.0 \mathrm{mM}$. 
To verify the ion selectivity of valinomycinmodified graphene FETs, $\mathrm{Na}$ ion concentration dependence of transfer characteristics in graphene FETs was investigated, as shown in Fig. 4. The transfer characteristics of valinomycin-modified graphene FETs were almost unchanged; almost no Dirac point shifts were found over the $\mathrm{Na}$ ion concentration range between 0 and $1.0 \mathrm{mM}$, indicating that the ISM membrane suppressed the nonspecific binding of nontarget cations to the surface of the graphene channels. Therefore, valinomycinmodified graphene FETs are useful to fabricate ion sensors with high sensitivity and selectivity.

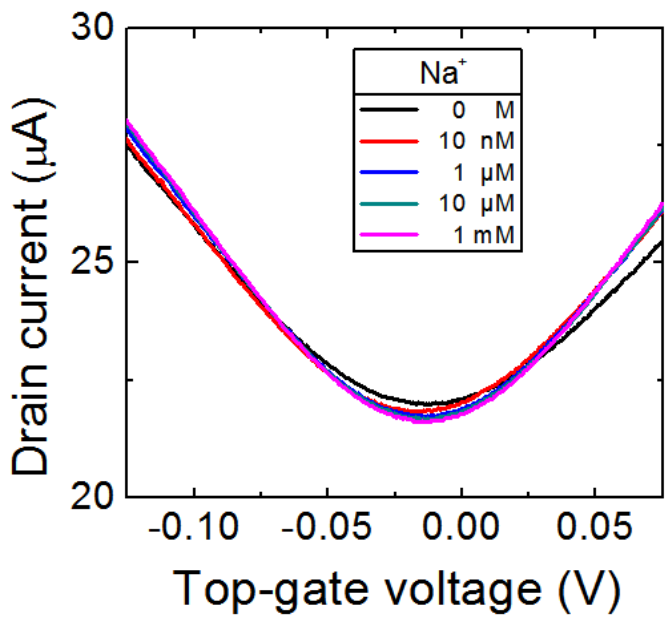

Fig. 4. Transfer characteristics of a valinomycinmodified graphene FET in the $100 \mathrm{mM}$ Tris- $\mathrm{HCl}$ buffer solution with various $\mathrm{Na}$ ion concentrations over the range from 0 to $1.0 \mathrm{mM}$.

\section{Conclusions}

We have demonstrated that graphene FETs modified with valinomycin are highly sensitive and selective for the electrical detection of $\mathrm{K}$ ions in the electrolyte. The $\mathrm{K}$ ion concentration dependence of the valinomycin-modified graphene FET transfer characteristics revealed that the transfer curves were negatively shifted as the $\mathrm{K}$ ion concentration increased, and a semilogarithmic dependence between the topgate voltage at the Dirac point and the $\mathrm{K}$ ion concentration was observed. Furthermore, the valinomycin-modified graphene FETs effectively detected $\mathrm{K}$ ions over a wide concentration range from $10 \mathrm{nM}$ to $1.0 \mathrm{mM}$. These observations can be explained by the accumulation of positive charge on the surface of the graphene channel, because of the specific binding of $\mathrm{K}$ ions to valinomycin in the ISM. In contrast, the valinomycin-modified graphene FET transfer characteristics showed no change when the concentration of $\mathrm{Na}$ ions was increased. These results indicate that the valinomycin-modified graphene FETs responded specifically to $\mathrm{K}$ ions in solution, and will prove useful for high sensitivity, selective ion sensors.

\section{Acknowledgements}

This research was partially supported by the New Energy and Industrial Technology Development Organization (NEDO), by the Core Research for Evolutional Science and Technology (CREST) from the Japan Science and Technology Agency (JST), by Management Expenses Grants for National University Corporations from the Ministry of Education, Culture, Sports, Science, and Technology of Japan, and by Japan Society for the Promotion of Science Core-to-Core Program. The authors are grateful for support from Dr. Shin-ichi Wakida from the National Institute of Advanced Industrial Science and Technology.

\section{References}

[1] E. Stern, J. F. Klemic, D. A. Routenberg, P. N. Wyrembak, D. B. Turner-Evans, A. D. Hamilton, D. A. LaVan, T. M. Fahmy, and M. A. Reed, Label-free immunodetection with CMOScompatible semiconducting nanowires, Nature 445, 519-522 (2007); doi: 10.1038/nature05498

[2] K. Maehashi, and K. Matsumoto, Label-Free Electrical Detection Using Carbon NanotubeBased Biosensors, Sensors 9, 5368-5378 (2009); doi: $10.3390 / \mathrm{s} 90705368$

[3] J. Okuno, K. Maehashi, K. Kerman, K. Matsumoto, Y. Takamura, and E. Tamiya, Labelfree immunosensor for prostate-specific antigen based on single-walled carbon nanotube arraymodified microelectrodes, Biosens. Bioelectron. 22, 2377-2381 (2007); doi:10.1016/j.bios.2006.09.038

[4] Y. Cui, Q. Wei, H. Park, C.M. Lieber, Nanowire Nanosensors for Highly Sensitive and Selective Detection of Biological and Chemical Species, Science 293, 1289-1292 (2001); doi: 10.1126/science. 1062711

[5] Y. Tsujita, K. Maehashi, K. Matsumoto, M. Chikae, S. Torai, Y. Takamura and E. Tamiya, Carbon Nanotube Amperometric Chips with Pneumatic Micropumps, Jpn. J. Appl. Phys. 47, 2064-2067 (2008); doi: 10.1143/JJAP.47.2064

[6] A. K. Geim and K. S. Novoselov, The rise of graphene, Nature Materials 6 183-191 (2007); doi: $10.1038 / n$ mat1849

[7] K. I. Bolotina, K. J. Sikesb, Z. Jianga, M. Klimac, G. Fudenberga, J. Honec, P. Kima, and H. L. Stormer, Ultrahigh electron mobility in suspended graphene, Solid State Communications 146, 351355 (2008); doi: 10.1016/j.ssc.2008.02.024

[8] Y. Ohno, K. Maehashi, Y. Yamashiro, and K. Matsumoto, Electrolyte-Gated Graphene FieldEffect Transistors for Detecting $\mathrm{pH}$ and Protein 
Adsorption, Nano Letters 9, 3318-3322 (2009); doi: $10.1021 / \mathrm{n} 1901596 \mathrm{~m}$

[9] Y. Sofue, Y. Ohno, K. Maehashi, K. Inoue, and K. Matsumoto, Highly Sensitive Electrical Detection of Sodium lons Based on Graphene Field-Effect Transistors, Japanese Journal of Applied Physics 50, 06GE07-1-4 (2011); doi: 10.1143/JJAP.50.06GE07

[10] S. Mao, K. Yu, G. Lu, and J. Chen, Highly Sensitive Protein Sensor Based on ThermallyReduced Graphene Oxide Field-Effect Transistor, Nano Research 4, 921-930 (2011); doi: $10.1007 / \mathrm{s} 12274-011-0148-3$

[11] Y. Ohno, K. Maehashi, and K. Matsumoto, LabelFree Biosensors Based on Aptamer-Modified Graphene Field-Effect Transistors, Journal of the American Chemical Society 132, 18012-18013 (2010); doi: 10.1021/ja108127r

[12] A. R. Lavinia, V. Pioda, and W. Simon, Highly Selective Potassium Ion Responsive LiquidMembrane Electrode, Anal. Lett. 2, 665-674 (1969); doi: 10.1080/00032716908051343

[13] K.-S. Chang, C.-J. Sun, P.-L. Chiang, A.-C. Chou, M.-C. Lin, C. Liang, H.-H. Hung, Y.-H. Yeh, C.-D. Chen, C.-Y. Pan, Y.-T. Chen, Monitoring extracellular $\mathrm{K}+$ flux with a valinomycin-coated silicon nanowire field-effect transistor, Biosens. Bioelectr. 31, 137-143 (2012); doi: 10.1016/j.bios.2011.10.005

[14] Y. Ohno, K. Maehashi, and K. Matsumoto, Chemical and biological sensing applications based on graphene field-effect transistors, Biosensors and Bioelectronics 26, 1727-1730 (2010); doi: 10.1016/j.bios.2010.08.001

[15] A. Ferrari, J. Meyer, V. Scardaci, C. Casiraghi, M. Lazzeri, F. Mauri, S. Piscanec, D. Jiang, K. Novoselov, S. Roth, and A. Geim, Raman Spectrum of Graphene and Graphene Layers, Physical Review Letters 97, 187401-1-4 (2006); doi: 10.1103/PhysRevLett.97.187401

[16] Y. Hakamata, Y. Ohno, K. Maehashi, K. Inoue, and K. Matsumoto, External-Noise-Induced Small-Signal Detection with Solution-Gated Carbon Nanotube Transistor, Appl. Phys. Exp. 4, 045102 (2011); doi: 10.1143/APEX.4.045102

[17] Y. Yamamoto, Y. Ohno, K. Maehashi, and K. Matsumoto, Noise Reduction of Carbon Nanotube Field-Effect Transistor Biosensors by Alternating Current Measurement, Jpn. J. Appl. Phys. 48, 06FJ01 (2009); doi: 10.1143/JJAP.48.06FJ01

[18] K. Maehashi, T. Katsura, K. Kerman, Y. Takamura, K. Matsumoto, and E. Tamiya, LabelFree Protein Biosensor Based on AptamerModified Carbon Nanotube Field-Effect Transistors, Analytical Chemistry 79, 782-787 (2007); doi: 10.1021/ac060830g

[19] T. Katsura, Y. Yamamoto, K. Maehashi, Y. Ohno and K. Matsumoto, High-Performance Carbon Nanotube Field-Effect Transistors with Local Electrolyte Gates, Jpn. J. Appl. Phys. 47, 20602063 (2008); doi: 10.1143/JJAP.47.2060 